\title{
Analysis of Primary Metabolites in Cabbage (Brassica oleracea var. capitata) Varieties Correlated with Antioxidant Activity and Taste Attributes by Metabolic Profiling
}

\author{
Ryota Mabuchi *(1), Mao Tanaka, Chihori Nakanishi, Nanako Takatani and Shota Tanimoto \\ Faculty of Human Culture and Science, Prefectural University of Hiroshima, 1-1-71, Ujina-Higashi, Minami-ku, \\ Hiroshima 734-8558, Japan; q821004tb@ed.pu-hiroshima.ac.jp (M.T.); open-goma@kmj.biglobe.ne.jp (C.N.); \\ q502020kq@ed.pu-hiroshima.ac.jp (N.T.); s-tanimoto@pu-hiroshima.ac.jp (S.T.) \\ * Correspondence: mabuchi@pu-hiroshima.ac.jp; Tel.: +81(82)-251-9767 \\ Academic Editors: Nikolaos S. Thomaidis and Marilena Dasenaki \\ Received: 28 October 2019; Accepted: 22 November 2019; Published: 25 November 2019

\begin{abstract}
Brassica vegetables, such as cabbage, have many health benefits arising from their antioxidant and anticancer properties. These properties are endowed by the metabolite composition of the plant, and it is therefore important to elucidate the metabolic profile and associated activities in this genus. This study objectively evaluated the characteristics of cabbage varieties using metabolic profiling to identify the primary metabolic components that correlate with antioxidant activity and taste attributes. GC-MS analysis was used to identify the primary metabolites. Antioxidant activity was measured by oxygen radical absorbance capacity (ORAC) and 2,2-diphenyl-1-picrylhydrazyl radical (DPPH) scavenging assays, and an electronic tongue was used to quantitate nine taste attributes. Orthogonal projections to latent structures (OPLS) using SIMCA 14 correlated the metabolite components with the taste and antioxidant characteristics. We identified 4-aminobutyric acid, fructose 1-phosphate, adipic acid, 5-oxoproline, $\mathrm{N}$-acetylglycine, $\mathrm{O}$-phosphoethanolamine, and homovanillic acid as important determinants of DPPH scavenging activity and umami, sourness, acidic bitterness, irritant and saltiness, bitterness, astringency, and richness, respectively. These metabolites represent markers indicating breed differences and contribute to differential cabbage functionality. These studies could be extended to measure additional metabolites, as well as to understand the role of growth conditions on the metabolic profile and health benefits of plants.
\end{abstract}

Keywords: cabbage; metabolomics; GC-MS; DPPH; ORAC; electronic tongue; OPLS

\section{Introduction}

Brassica vegetables, including cabbage, broccoli, and cauliflower, are widely eaten around the world as both fresh produce and processed products. These vegetables contain unique compounds that provide them with distinctive tastes, nutritional values, and health applications. The flavor of Brassica vegetables has been attributed to isothiocyanates generated from the hydrolysis of glucosinolates $[1,2]$. Both the glucosinolates and isothiocyanates are vital nutrients that have been associated with the prevention of cancer $[2,3]$. These vegetables are also reported to have a high concentration of antioxidant compounds, such as phenols and flavonoids $[4,5]$. Owing to the clear benefits provided by their consumption, a complete library of the range of components in this plant genus would be of great use for a variety of biological and biochemical industries.

Food metabolomics, a key technique used in foodomics, is the best way to identify characteristic metabolic components among distinct crop varieties [6-9]. Multiple chemical components of Brassica 
have already been characterized through metabolomics [9-16]. Kim et al. were able to distinguish Chinese and Korean cabbages using proton nuclear magnetic resonance $\left({ }^{1} \mathrm{H}-\mathrm{NMR}\right)$ spectroscopy [10]. Jeon et al. differentiated the primary and secondary metabolic components of green and purple pak choi using gas chromatography-time-of-flight mass spectrometry (GC-TOF-MS) and high- performance liquid chromatography-ultraviolet (HPLC-UV) detection [11]. However, a correlation between these characteristic components and the taste and antioxidant properties of the vegetables has not yet been determined.

In-depth studies of cabbage (Brassica oleracea) would be highly informative, as a wide variety of subspecies has been created worldwide through extensive breeding and crossbreeding. For example, Japan has multiple cabbage varieties grown in different places, during distinct seasons, and for specific uses (raw, cooked, and processed). These varieties display unique appearances and disease resistance and thus can also be expected to differ in their taste and metabolic compositions. However, an accurate characterization of the components in these cabbage varieties is far from complete.

This study used metabolic profiling to characterize the antioxidant activity and taste attributes of six Japanese cabbage varieties. Water-soluble primary metabolic components were identified by GC-MS analysis. The antioxidant activity and taste attributes were measured using biochemical assays and an electronic tongue, respectively. Multivariate analysis of these data identified 4-aminobutyric acid, fructose 1-phosphate, adipic acid, 5-oxoproline, $\mathrm{N}$-acetylglycine, $\mathrm{O}$-phosphoethanolamine, and homovanillic acid as potential breed-specific components underlying the antioxidant activity and taste of each variety.

\section{Results and Discussion}

\subsection{Annotated Water-Soluble Primary Metabolites by GC-MS Analysis}

Six varieties of Japanese cabbage were evaluated in this study: YR-ginjiro (Y), Satou-kun (S), Okina (O), Kinkei-201go (KI), Kogetsu-SP (KO), and Hiro-kanran (H). GC-MS was used to obtain the total ion chromatograms (TICs) for each variety (Figure S1). Annotation of the TIC peaks revealed the presence of 331 unique metabolites (Table S1). The number of annotated metabolites by cabbage variety was: $301(\mathrm{Y}), 278(\mathrm{~S}), 284(\mathrm{O}), 281(\mathrm{KI}), 287(\mathrm{KO})$, and $274(\mathrm{H})$. The majority of these primary metabolites were amino acids, organic acids, and carbohydrates. Although some of these compounds resembled primary metabolites found during earlier metabolomics studies of Brassica [11,17,18], these previous studies only identified about 40-50 primary metabolic components $[11,18]$. Previous studies had used GC-OF-MS for qualitative and quantitative metabolic profiling; however, the peaks in our work were detected using the retention index, and the metabolic components were comprehensively annotated based on their match rate with the commercially available GC/MS Metabolite Component Database (Ver. 2, Shimadzu). This metabolic fingerprinting method allowed for the identification of a far greater number of metabolic components, a desirable feature when attempting to correlate a chemical profile with the complex activities and attributes of an organism.

\subsection{In Vitro Antioxidant Assay of DPPH and ORAC}

Established methods exist for measuring the antioxidant activity of food and other agricultural products. These methods generally use either hydrogen atom transfer (HAT) or single electron transfer (SET) for detecting antioxidant activity, and it is ideal to use both techniques to ensure an accurate characterization $[19,20]$. In our study, the antioxidant activity was measured using oxygen radical absorbance capacity (ORAC) and 2,2-diphenyl-1-picrylhydrazyl radical (DPPH) scavenging activity as the HAT- and SET-based techniques, respectively (Table S1). The average antioxidant activity of the six cabbage varieties measured by DPPH scavenging and ORAC was $6.21 \pm 1.37$ and $14.6 \pm 0.92$ activity, respectively, which are similar to previously reported values $[18,21]$. A one-way analysis of variance (ANOVA) test was used to compare the antioxidant activities of the cabbage varieties (Figure 1). While there was no significant difference observed between the varieties using ORAC ( $p=$ 
0.463, Figure 1B), the antioxidant activity of the different breeds varied significantly during DPPH scavenging $(p<0.05$, Figure 1A). These differences were further confirmed using Tukey's multiple comparison procedure. From these analyses, it could be concluded that cabbage variety $\mathrm{H}$ showed the highest antioxidant activity during DPPH scavenging, while the lowest activity was observed for $O$. The different antioxidant activities observed for the cabbage varieties within and between the two tests are reflective of the different antioxidant mechanisms measured by the assays [21,22]. As such, it appears that the six cabbage varieties have similar antioxidants or antioxidant mechanisms with respect to ORAC, but unique compounds or pathways for DPPH scavenging.
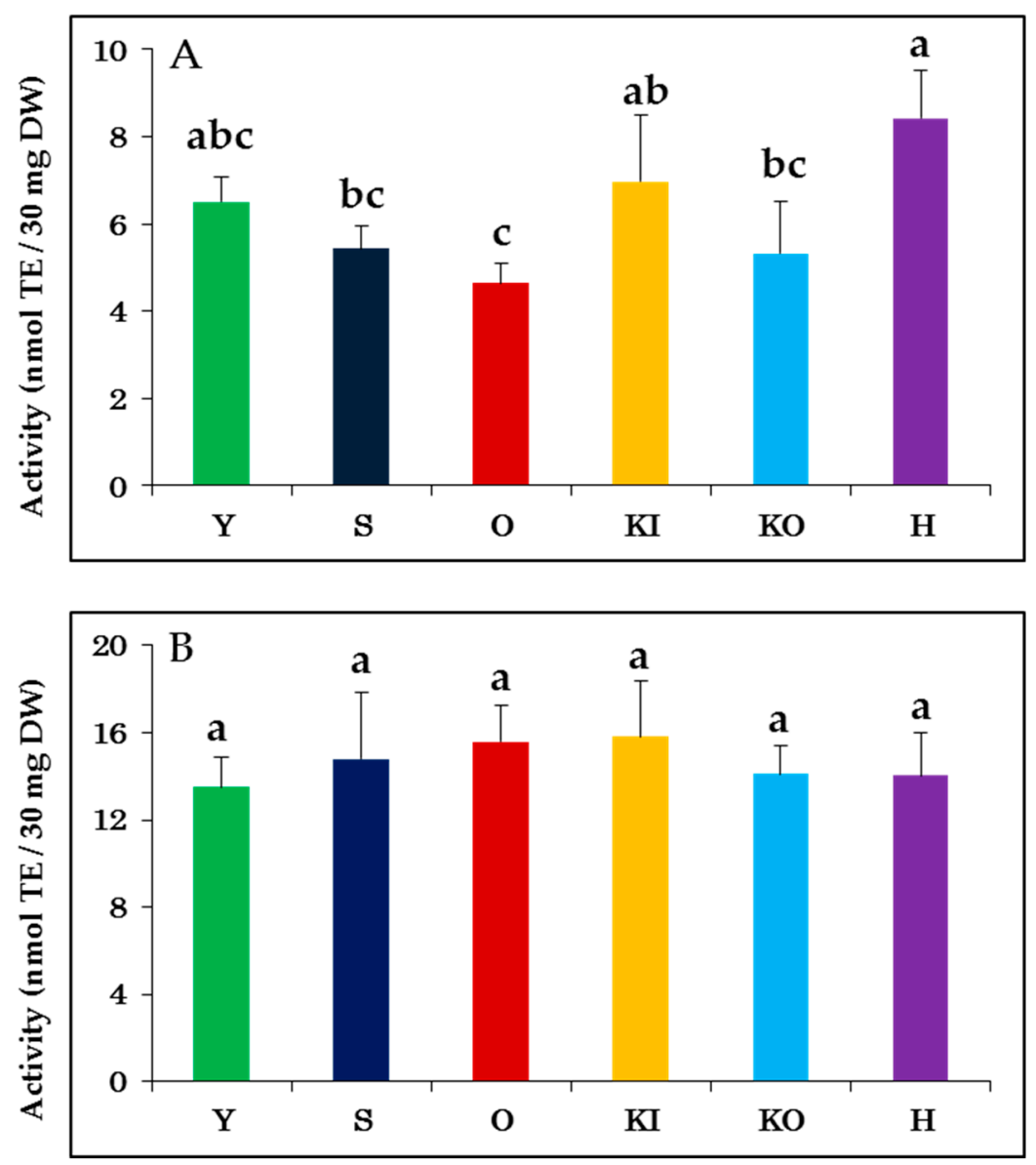

Figure 1. ANOVA test of antioxidant activity measured by (A) DPPH scavenging and (B) oxygen radical absorbance capacity (ORAC). Y: YR-Ginjiro, S: Satou-kun, O: Okina, KI: Kinkei-201go, KO: Kogetsu-SP, H: Hiro-kanran. TE: Trolox equivalent; DW: Dry weight of sample. The values are mean \pm SD $(n=5)$. Significant differences $(p<0.05)$ between the cabbage varieties are indicated by different letters. The $p$-values between each group are shown in Table S2.

\subsection{Measurement of Taste Attributes by Electronic Tongue}

The traditional method for evaluating the taste of foods, the sensory test, involves an experienced evaluator (called a sensory panelist) tasting the sample. However, this method suffers from low objectivity and reproducibility and requires substantial labor from the panelists and their trainers. The electronic tongue functions as an alternative to this process; it can be used to more objectively identify and quantify tastes [23] and has already been successfully applied to a variety of foods [23-25]. There are a small, but growing, number of reported applications of the electronic tongue to Brassica vegetables, including a recent study on the taste evaluation of broccoli flowers [26].

We used the electronic tongue to quantify the different tastes among the cabbage varieties (Table S1). Of the nine taste attributes measured, differences among the varieties were detected for all tastes 
except richness (Figure 2). H had the lowest sourness value and was significantly different from KI, $\mathrm{KO}$, and $\mathrm{Y}$, which had the highest value (Figure 2A). Y, KO, and $\mathrm{O}$ were significantly different in acidic bitterness, with $\mathrm{O}$ having the highest and $\mathrm{Y}$ the lowest acidic bitterness value (Figure 2B). Most of the varieties were distinct in irritant measurements, with $\mathrm{Y}$ and $\mathrm{KO}$ showing the highest values, while $\mathrm{H}, \mathrm{S}$, and KI showed negative values for this attribute (Figure 2C). H showed the highest umami value and was significantly different from the other varieties, except for S (Figure 2D). Saltiness distinguished $\mathrm{Y}$ and $\mathrm{KO}$ from each other and from the other four varieties (Figure 2E). H was the least bitter and astringent variety, differing significantly from all other varieties in both of these tastes (Figure 2F,G). Astringency also varied significantly between groups of " $\mathrm{Y}, \mathrm{O}, \mathrm{KO}$ " and "S, KI" (Figure 2G). $\mathrm{H}$ and KI were the least sweet cabbage varieties, while $Y$ was significantly sweeter than all other varieties (Figure 2I). These results show the utility of the electronic tongue to quantitatively evaluate the tastes of cabbage varieties.
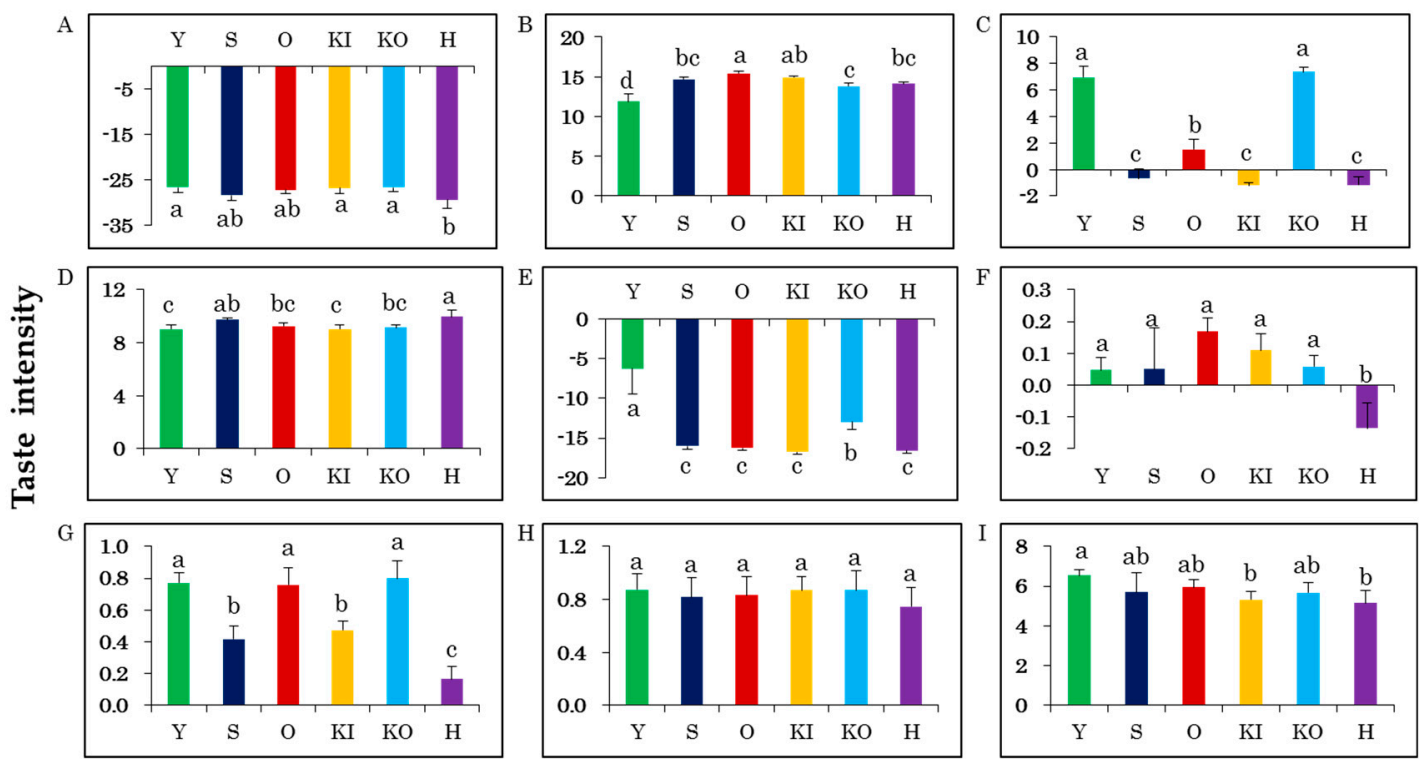

Figure 2. Taste attribute measurements of cabbage varieties using an electronic tongue. (A) Sourness, (B) acidic bitterness, (C) irritant, (D) umami, (E) saltiness, (F) bitterness, (G) astringency, $(\mathbf{H})$ richness, (I) sweetness. The values are mean $\pm \mathrm{SD}(n=5)$. Significant differences $(p<0.05)$ between the cabbage varieties are indicated by different letters. The $p$-values between each group are shown in Table S2.

\subsection{Metabolic Profiling of Varieties by Principal Component Analysis (PCA) and OPLS-Discriminant Analysis (DA)}

A data set was created consisting of the metabolic components as $x$ variables, and the antioxidant and taste values as $y$ variables. This data set was subjected to statistical analysis using SIMCA 14 (MKS Instruments). We initially performed PCA-X analysis on the metabolites to gain an overview of the cabbage varieties (Figure S2). However, the relationship between the metabolites and the cabbage varieties was unclear. Therefore, an OPLS-DA analysis was performed for better discrimination. Numbers 1-6 were assigned to each variety and a discriminant analysis was performed in OPLS-DA (Figure S3). This was autofitted with a $3+1+0$ model and provided an R2Y $=0.524$ and Q2 $=0.365$, indicating that the model was not significant $(p=0.91)$. H was separated from the other varieties in component $1(\mathrm{t}[1])$, while component $2(\mathrm{t}[2])$ clustered $\mathrm{KI}, \mathrm{O}$, and $\mathrm{S}$ in the positive direction and $\mathrm{Y}$ and $\mathrm{KO}$ in the negative direction (Figure 3A). Varieties that showed similar trends were grouped and reanalyzed by OPLS-DA (group 1: $\mathrm{H}$, group 2: $\mathrm{KI}, \mathrm{O}$, and $\mathrm{S}$, group 3: $\mathrm{KO}$ and $\mathrm{Y}$ ). This model was autofitted as $2+1+0$ with $\mathrm{R} 2 \mathrm{Y}=0.867$ and $\mathrm{Q} 2=0.798$, indicating a significant model $(p<0.05)$. This model gave an R2Y intercept $=0.584$ and a $\mathrm{Q} 2$ intercept $=-0.343$ in a model accuracy by permutation test $(n=200)$. While the R2Y intercept was somewhat high, the Q2 intercept was less than 0 , confirming 
some degree of model accuracy. In particular, $\mathrm{H}$ was distinct from the other varieties, likely reflecting that it is a wild species long cultivated in Japan, whereas the other varieties have been crossbred. These genetic differences are likely expressed through the metabolic profile. On the other hand, groups 2 (KI, $\mathrm{O}$, and $\mathrm{S}$ ) and 3 (KO and $\mathrm{Y}$ ) differ in their cultivation areas, which have been shown to affect metabolites in cabbage plants [10]. This is likely due to differences in soil nutrients present in the cultivation fields.
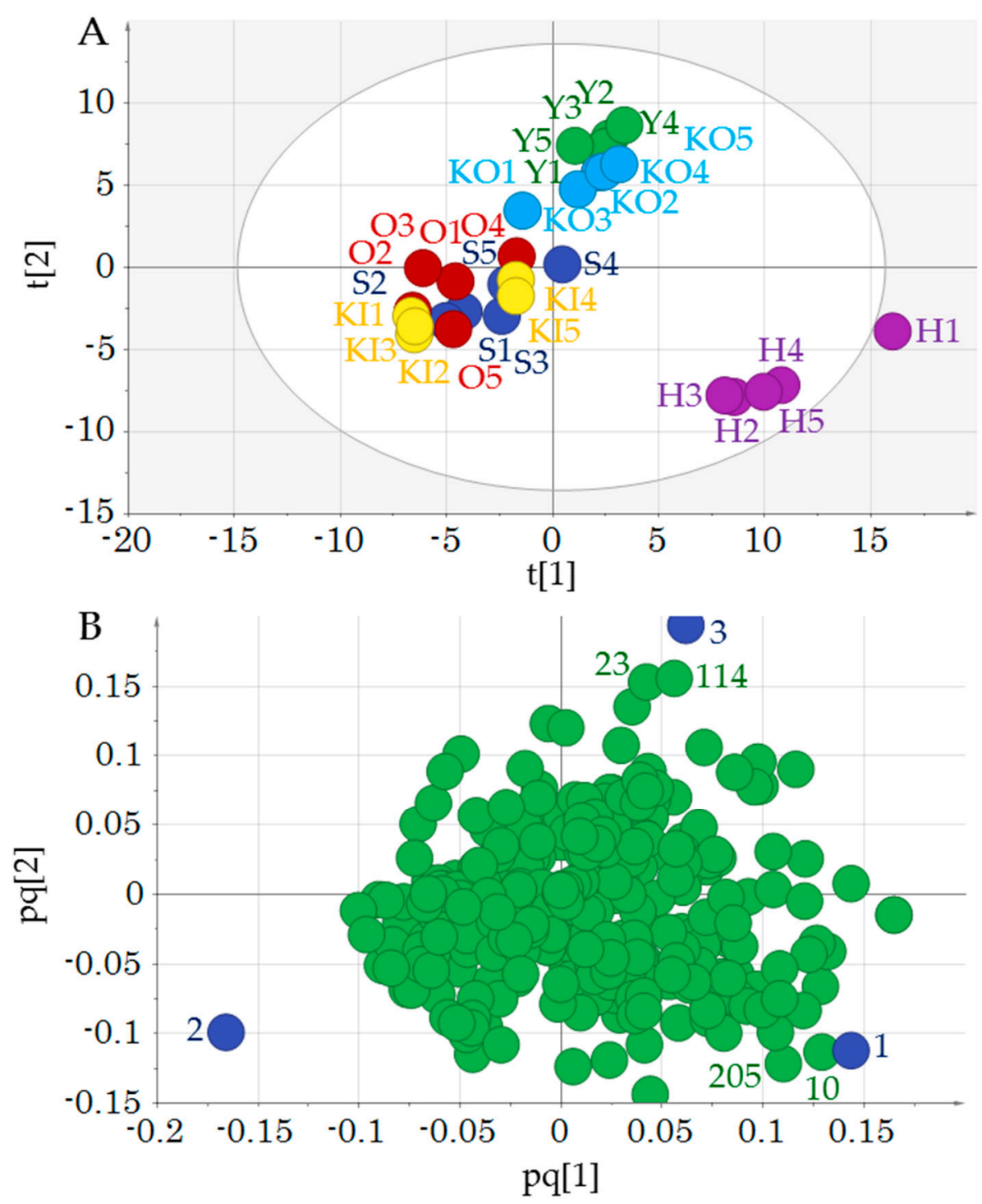

Figure 3. Orthogonal projections to latent structures-discriminant analysis (OPLS-DA) of cabbage variety metabolites. (A) Score plots and (B) loading plots for the three cabbage groups identified during the analysis (group 1: H; group 2: KI, O, and S; group 3: KO and Y). Letters and numbers in (A) indicate sample ID and numbers in (B) indicate group identity (blue circle) (Table S1). Numbers adjacent to green circles correspond to $x$ variable IDs in Table S1: Alanine (10), 3-hydroxyisobutyric acid (23), 5-oxoproline (114), and O-phosphoethanolamine (205). Cross-validated (CV)-ANOVA was used to calculate the $p$-value of this model $(p=0.00000046)$.

The metabolites contributing to these varietal differences are shown in the loading plot (Figure 3B). The components that correlated with $\mathrm{H}$ were $\mathrm{O}$-phosphoethanolamine and alanine. $O$-phosphoethanolamine is a metabolite involved in the synthesis of phosphatidylcholine, the main phospholipid of eukaryotic cells in plants [27]. Alanine is an amino acid that exhibits sweetness and umami. Studies evaluating free amino acids among inbred varieties of cabbage have shown varietal 
differences in alanine content [28]. Group 3 (KO and Y) was correlated with 3-hydroxyisobutyric acid and 5-oxoproline. The compound 3-hydroxyisobutyric acid is a metabolic intermediate of valine, and 5-oxoproline is a derivative amino acid of glutamine. Both are metabolites involved in amino acid biosynthesis. Metabolites may be associated with physiological differences between plant varieties; however, it is difficult to ascribe a specific function to these metabolite differences. Nevertheless, metabolites can serve as characteristic markers of cabbage varieties.

PCA-Y was then performed to reveal the relationship between the $y$ variables and the varieties. In the score plots (Figure $4 \mathrm{~A}$ ), the first principal component separates $\mathrm{H}$ in the positive direction from $\mathrm{Y}$ and $\mathrm{KO}$ in the negative direction. $\mathrm{KI}$ and $\mathrm{O}$ cluster together in the second principal component in the positive direction separately from $\mathrm{H}$ and $\mathrm{Y}$ in the negative direction. $\mathrm{H}$ is correlated with umami and DPPH scavenging, and $Y$ is correlated with irritant and saltiness taste attributes (Figure 4B). Differences in the cabbage variety and cultivation area were reflected in taste attributes and antioxidant properties, as was observed with the metabolic profile. For example, KO and Y are cultivated in the same soil and cluster together in our analysis, indicating that the soil used for cultivation likely affects the taste and antioxidant properties of cabbage. This association will require further verification.

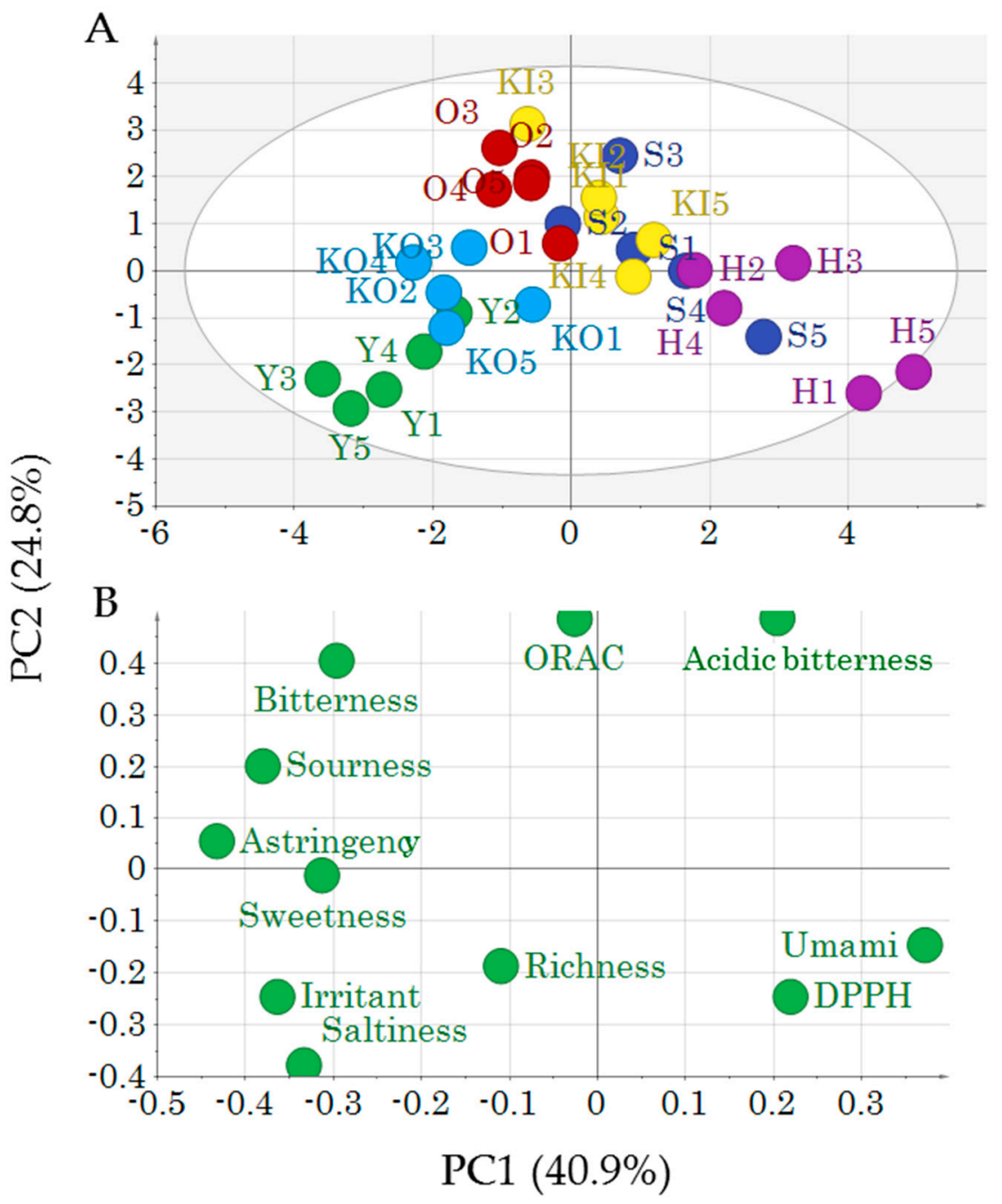

Figure 4. (A) Score plots and (B) loading plots of principal component analysis (PCA)-Y results for the different cabbage varieties. Letters and numbers in (A) indicate sample ID (Table S1).

In Brassica plants, such as cabbage, the compounds responsible for bitter and irritant taste attributes are isothiocyanates and glucosinolate [29], while ascorbic acid and phenol components are known to provide antioxidant activity targeting DPPH [30-32]. These are often characterized as secondary 
metabolic components of plants. In our work, variety $\mathrm{Y}$ has a strong irritant taste and would therefore be expected to contain more isothiocyanates and glucosinolate than other varieties. Likewise, $\mathrm{H}$, which has a high antioxidant activity against DPPH, should have higher antioxidant content. However, these secondary metabolic components were not investigated in this study but will provide avenues for future analyses.

\subsection{OPLS Correlation Analysis of Metabolic Profiling with Antioxidant Activity and Taste Attributes}

OPLS analysis was performed for each $y$ variable to identify the metabolites correlated with these specific characteristics (Table 1). Statistically significant models could be created for all $y$ variables, except ORAC and sweetness. The relationship between the variables and the different cabbage varieties could be visualized by regression analysis of each $y$ variable and the predicted value from the corresponding model (Figure 5). From these analyses, H could be positively correlated with DPPH activity and umami and negatively correlated to sourness, astringency, and bitterness. Y shows a positive correlation with saltiness and a negative correlation with acidic bitterness. Irritant was positively correlated only with $\mathrm{KO}$ and $\mathrm{Y}$. Although a significant predictive model could be created for richness, no differences between the varieties were observed.

Table 1. Evaluation of the models obtained from the OPLS analysis of each $y$ variable.

\begin{tabular}{|c|c|c|c|c|c|c|c|c|}
\hline \multirow[b]{2}{*}{ y Variable } & \multirow[b]{2}{*}{$\mathbf{A}^{1}$} & \multirow[b]{2}{*}{$\mathrm{N}^{2}$} & \multirow[b]{2}{*}{ R2X(cum) } & \multirow[b]{2}{*}{ R2Y(cum) } & \multirow[b]{2}{*}{ Q2(cum) } & \multirow[b]{2}{*}{$p$-value ${ }^{3}$} & \multicolumn{2}{|c|}{ Permutation Test } \\
\hline & & & & & & & $\begin{array}{c}\mathrm{R} 2 \mathrm{Y} \\
\text { Intercept }\end{array}$ & $\begin{array}{c}\mathrm{Q} 2 \\
\text { Intercept }\end{array}$ \\
\hline $\mathrm{DPPH}$ & $1+1+0$ & 30 & 0.233 & 0.866 & 0.595 & 0.016 & 0.668 & -0.294 \\
\hline ORAC & $1+1+0$ & 30 & 0.197 & 0.770 & 0.421 & 0.26 & - & - \\
\hline Sourness & $1+2+0$ & 30 & 0.319 & 0.957 & 0.839 & 0.000015 & 0.702 & -0.355 \\
\hline Acidic bitterness & $1+1+0$ & 30 & 0.215 & 0.874 & 0.684 & 0.0010 & 0.679 & -0.314 \\
\hline Irritant & $1+2+0$ & 30 & 0.308 & 0.977 & 0.869 & 0.0000045 & 0.685 & -0.367 \\
\hline Umami & $1+2+0$ & 30 & 0.305 & 0.961 & 0.858 & 0.0000046 & 0.714 & -0.294 \\
\hline Saltiness & $1+2+0$ & 30 & 0.32 & 0.947 & 0.792 & 0.00011 & 0.729 & -0.358 \\
\hline Bitterness & $1+1+0$ & 30 & 0.242 & 0.85 & 0.575 & 0.022 & 0.681 & -0.321 \\
\hline Astringency & $1+2+0$ & 30 & 0.287 & 0.975 & 0.869 & 0.000011 & 0.696 & -0.332 \\
\hline Richness & $1+1+0$ & 30 & 0.259 & 0.866 & 0.746 & 0.000035 & 0.668 & -0.334 \\
\hline Sweetness & $1+1+0$ & 30 & 0.226 & 0.835 & 0.472 & 0.20 & - & - \\
\hline
\end{tabular}

${ }^{1} \mathrm{~A}=$ number of models. ${ }^{2} \mathrm{~N}=$ number of samples used in producing the models. ${ }^{3} p$-values were obtained from the analysis of cross-validated predictive residuals (CV-ANOVA).

Values for the $x$ variables important for prediction (VIP) were calculated for the $y$ variables for which it was possible to create significant models and observe varietal differences. A VIP value of 1.0 or higher is considered to indicate an important $x$ variable for the model [33], which varies between cabbage varieties. Many metabolites showed VIP values of 1.0 or more (Table S3). The metabolites showing the highest VIP values for their respective $y$ variables are: 4-aminobutyric acid (DPPH and umami), fructose 1-phosphate (sourness), adipic acid (acidic bitterness), 5-oxoproline (irritant and saltiness), $\mathrm{N}$-acetylglycine (bitterness), O-phosphoethanolamine (astringency), and homovanillic acid (richness). These primary metabolic components are considered candidate markers for each breed that provide the basis for its characteristic taste and antioxidant activity.

In this metabolomic study, electronic tongue and in vitro antioxidant assays were used to evaluate the differences in primary metabolites between cabbage varieties. These primary metabolites were correlated to the taste attributes and antioxidant activity of the cabbages. Previous studies in microalgae, Prunus dulcis leaves, and Neptunia oleracea [34-36] evaluated the correlation between antioxidant activity and metabolites based on metabolomics alone. Japanese sake, coffee, and fish have been analyzed using the electronic tongue, as well as metabolomic measurements [37-40]. This study successfully extends these methods into cabbage varieties to correlate their metabolite content with their characteristic taste 
and antioxidant activities. This approach could be extended to additional cabbage varieties and for evaluating the specific characteristics of individual cabbage varieties.
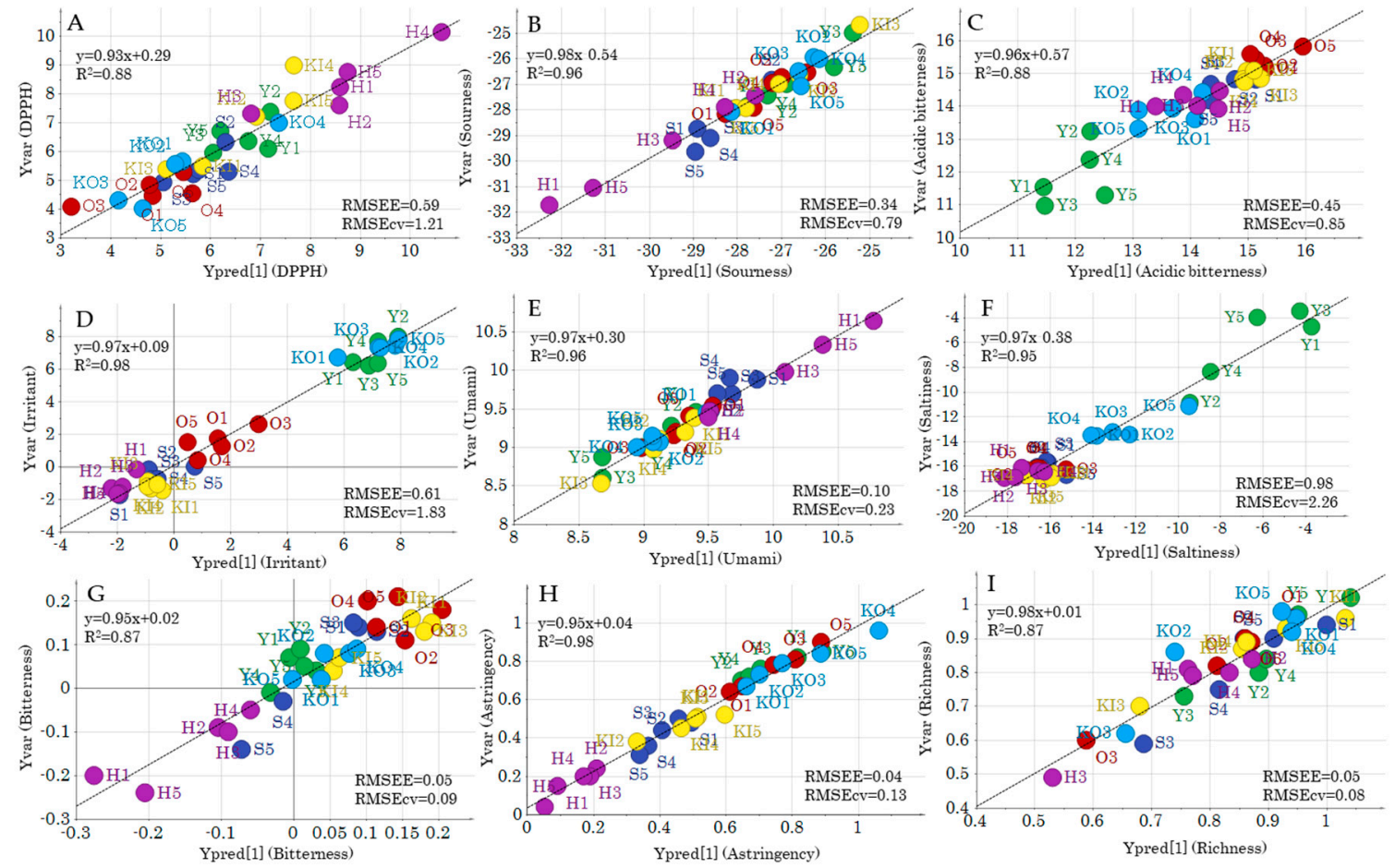

Figure 5. Regression analysis of the OPLS predictive model for each $y$ variable. (A) DPPH, (B) sourness, (C) acidic bitterness, (D) irritant, (E) umami, (F) saltiness, (G) bitterness, (H) astringency, (I) richness. Alphanumeric labels indicate the sample IDs (Table S1). RMSEE: Root mean square errors of estimation; RMSEcv: Root mean square errors of cross-validation.

\section{Materials and Methods}

\subsection{Chemicals}

All reagents were obtained from commercial sources and were of guaranteed reagent. Methanol, chloroform, pyridine, and ribitol for GC-MS analysis were purchased from Wako Pure Chemicals Industries, Ltd. (Osaka, Japan). Methoxyamine hydrochloride and $N$-methyl-N-(trimethylsilyl)trifluoroacetamide (MSTFA) for GC derivatization were purchased from Sigma-Aldrich (St. Louis, MO, USA) and GL Sciences (Tokyo, Japan), respectively. DPPH and 2-morpholinoethanesulfonic acid (MES) for measuring DPPH activity were purchased from Sigma-Aldrich and TCI (Tokyo, Japan), respectively. Fluorescein and 2,2'-azobis(2-amidinopropane) dihydrochloride for use in the ORAC assay were purchased from Sigma-Aldrich and Wako, respectively. Trolox (Sigma-Aldrich) was used as a standard for DPPH and ORAC assays.

\subsection{Experimental Samples}

Six varieties of cabbage (H, O, KI, S, KO, and Y) cultivated in Kure City, Hiroshima Prefecture, Japan were used for this study. All varieties were harvested in December 2017, when they reached approximately equal maturity. $\mathrm{H}, \mathrm{O}, \mathrm{KI}$, and $\mathrm{S}$ were cultivated in the same field, and $\mathrm{KO}$ and $\mathrm{Y}$ were cultivated in other fields in the same region. $\mathrm{H}$ has long been cultivated in Hiro-Machi, Kure City, Hiroshima Prefecture. As $\mathrm{H}$ has not been crossbred with other varieties, it closely resembles the wild variety, native species cultivated in Japan from an earlier period. KO is bright green, harvested in winter, and has resistance towards diseases such as yellow dwarf disease. KI is an extremely fast-growing variety, and the head shows excellent hypertrophy. $\mathrm{O}$ is one of the representative cabbage 
varieties in Japan. It is resistant to yellow dwarf disease and relatively fast growing. The head is shaped like an oblate spheroid and has excellent shape stability. S has slightly larger outer leaves, a slightly flat head containing yellow leaves, and no anthocyanin. Y has dark green leaves and can be harvested in winter. Five heads of each variety were used for this work. The samples were prepared as follows: Approximately two outer leaves were peeled from the head, the remaining parts were cut into halves, and the inner core portion of the head was removed. The sample was then thinly chopped with a cabbage slicer (RCS-70, Happy Japan, Yamagata, Japan), put in a bag with a chuck, and stored at $-80{ }^{\circ} \mathrm{C}$ prior to analysis.

\subsection{GC-MS Analysis}

Details for GC-MS sample preparation and analysis have been described in our previous reports [39-41]. A portion of the sample stored at $-80{ }^{\circ} \mathrm{C}$ was lyophilized (FDU-1200, EYELA, Tokyo, Japan) overnight and then ground to a powder using a mill (TUBE-MILLC S001, IKA, Staufen, Germany) for $30 \mathrm{~s}$ at 25,000 rpm. Mixed solutions of methanol/ultrapure water/chloroform (2.5/1/1 $v / v / v, 1 \mathrm{~mL}$ ) and ribitol (internal standard, $0.2 \mathrm{mg} / \mathrm{mL}, 60 \mu \mathrm{L}$ ) were added to $50 \mathrm{mg}$ of the powdered sample. After stirring for $5 \mathrm{~min}$, the mixture was centrifuged $\left(16000 \times g, 0{ }^{\circ} \mathrm{C}, 5 \mathrm{~min}\right)$. Ultrapure water $(400 \mu \mathrm{L})$ was added to $800 \mu \mathrm{L}$ of the supernatant (methanol phase), which was then stirred for $1 \mathrm{~min}$ and centrifuged $\left(16000 \times g, 0{ }^{\circ} \mathrm{C}, 5 \mathrm{~min}\right)$. A $400 \mu \mathrm{L}$ portion of the supernatant was concentrated for $1 \mathrm{~h}$ using a centrifugal evaporator (CVE-2000, Eyela, Japan) and freeze-dried overnight. Oxime formation was performed by reacting the freeze-dried sample with pyridine-solubilized methoxyamine hydrochloride $(20 \mathrm{mg} / \mathrm{mL}, 50 \mu \mathrm{L})$ at $30^{\circ} \mathrm{C}$ for $90 \mathrm{~min}$. Trimethylsilylation was then achieved by treating the sample with MSTFA $(100 \mu \mathrm{L})$ at $37^{\circ} \mathrm{C}$ for $30 \mathrm{~min}$.

The derivatized samples were analyzed by GC-MS using a GCMS-QP2010 Ultra system (Shimadzu Co., Kyoto, Japan) equipped with an Agilent J\&W DB-5 column (length $30 \mathrm{~m}$, internal diameter $0.25 \mathrm{~mm}$, film thickness $1.00 \mu \mathrm{m}$, Agilent Technologies, Santa Clara, CA, USA). The GC oven temperature started at $100{ }^{\circ} \mathrm{C}$, held for $4 \mathrm{~min}$, increased to $320^{\circ} \mathrm{C}$ at $10^{\circ} \mathrm{C} / \mathrm{min}$, and held for $11 \mathrm{~min}$. The injection port temperature was $280^{\circ} \mathrm{C}$. The derivatized sample $(1 \mu \mathrm{L})$ was injected in split injection mode with a split ratio of 10:1. Helium was employed as the carrier gas at constant linear velocity of $39.0 \mathrm{~cm} / \mathrm{s}$, and the purge flow rate was $5 \mathrm{~mL} / \mathrm{min}$. Quadrupoles were used for MS mass separation, and electron impact was used for ionization. The ion source temperature was $200^{\circ} \mathrm{C}$, the interface temperature was $280{ }^{\circ} \mathrm{C}$, and the ionization voltage was $70 \mathrm{eV}$. Measurements were carried out in scan mode in the range of 45-600 m/z (unit mass resolution). Retention time correction of peaks (retention index) was carried out based on the retention time of a standard alkane series mixture (C-6 to C-33) using the automatic adjustment of retention time function of the Shimadzu GCMS solution software. Peak annotation was performed using the commercially available GC/MS Metabolite Component Database Ver. 2 (Shimadzu Co., Kyoto, Japan), which contains a mass spectral library. Peaks were annotated under the condition of possessing a similarity index of more than 80 and a target ion with a confirmation ion ratio of $\geq 50 \%$ in absolute tolerance.

\subsection{Antioxidant Assay}

DPPH scavenging activity was measured using the method described by Katsube et al. [42], with modifications. Briefly, a mixture of $30 \mathrm{mg}$ of the powdered sample (from the sample prepared for GC-MS) in $80 \%$ ethanol $(1 \mathrm{~mL})$ was vortexed, sonicated, and centrifuged $\left(10,000 \times g, 4{ }^{\circ} \mathrm{C}, 5 \mathrm{~min}\right)$. The supernatant was collected, and the resulting pellet was extracted an additional two times using this procedure. The supernatants were combined and $10 \mu \mathrm{L}$ of this solution was added to the wells of a 96-well plate and mixed with $200 \mu \mathrm{L}$ of DPPH working solution (0.4 mM DPPH ethanol solution/400 mM MES buffer solution/ultrapure water). The absorbance of the mixture was recorded at $520 \mathrm{~nm}$ within $10 \mathrm{~min}$ of mixing using a microplate reader (SpectraMax 340PC384, MOLECULAR DEVICES, Tokyo, Japan). DPPH was expressed as $\mu \mathrm{mol}$ Trolox equivalent (TE)/30 mg sample, using Trolox as the standard. 
ORAC was measured using the method described by Prior et al. [43], with modifications. A 150X dilution of the $80 \%$ ethanol extract from the DPPH measurements was prepared. The diluted sample solution $(25 \mu \mathrm{L})$ and $81.6 \mathrm{nM}$ fluorescein $(100 \mu \mathrm{L})$ were added to the wells of a 96-well plate. After shaking at $1200 \mathrm{rpm}$ for $10 \mathrm{~s}$, the plate was incubated for $30 \mathrm{~min}$ at $37^{\circ} \mathrm{C}$. A solution of 2,2'-azobis(2-amidinopropane) dihydrochloride $(200 \mathrm{mM}, 25 \mu \mathrm{L}$ ) was added to the wells, and the plate was shaken at $1200 \mathrm{rpm}$ for $10 \mathrm{~s}$. The fluorescence intensity (ex/em: $485 / 528 \mathrm{~nm}$ ) was then recorded 50 times at 2 min intervals using a microplate fluorometer (Fluoroskan Ascent FL, Thermo Fisher Scientific, Waltham, MA, USA). The area under the curve (AUC) in the spectra was calculated, and the ORAC value was expressed in nmol Trolox equivalent (TE)/30 $\mathrm{mg}$ sample, using Trolox as the standard.

The SPSS STATISTICS 24 software (IBM, Armonk, NY, USA) was used for statistical analysis. One-way ANOVA was used to compare the mean DPPH and ORAC activity values between the cabbage varieties. Significant differences found in antioxidant activity were subsequently tested by Tukey's multiple comparison test. The significance level was set at $5 \%(p<0.05)$.

\subsection{Electronic Tongue Measurement}

The electronic tongue measurements were performed using a previously reported method $[39,40]$, with modifications. Briefly, chopped cabbage stored at $-80^{\circ} \mathrm{C}(50 \mathrm{~g})$ was added to ultrapure water (250 mL) and homogenized with an ACE Homogenizer AM-7 (Nihonseiki Kaisha Ltd., Tokyo, Japan) at $5000 \mathrm{rpm}$ for $5 \mathrm{~min}$ over ice. After centrifugation $\left(15,000 \times g, 4{ }^{\circ} \mathrm{C}, 15 \mathrm{~min}\right), 100 \mathrm{~mL}$ of the supernatant was collected and diluted to $400 \mathrm{~mL}$. This solution was used to measure the initial taste and aftertaste of the cabbage.

The taste was measured using a TS-5000Z taste sensor system (Insent, Kanagawa, Japan). Each sample solution was tested using six sensor types: AAE (umami), CT0 (saltiness), CA0 (sourness), C00 (acidic bitterness), AE1 (irritant), and GL1 (sweetness). The differences in human perception of taste intensity were estimated using Weber's law from an average of three repeated measurements, and the resultant value was taken as the intensity of each taste attribute. The system detects two types of tastes, initial taste and aftertaste. In this study, the relative potentials obtained from each sensor probe were used to measure the selected initial tastes. The changes in membrane potential caused by adsorption for C00 (bitterness), AE1 (astringency), and AAE (richness) sensor probes were used to measure the aftertastes [44]. SPSS STATISTICS 24 was used for statistical analysis. One-way ANOVA was used to compare the mean values between cabbage varieties for each taste attribute. Attributes that showed significant differences were subsequently tested by Tukey's multiple comparison test. The significance level was set at $5 \%(p<0.05)$.

\subsection{Multivariate Analysis}

SIMCA 14 (MKS Instruments, Andover, MA, USA) was used for multivariate analysis. The results were evaluated as described by Eriksson et al. [33]. The data sets consisted of the $x$ variables (the intensity of each annotated metabolite) and the $y$ variables (measured values of each taste attribute and two antioxidant activities). PCA-X or Y, the unsupervised learning analysis without the $y$ or $x$ variables, respectively, was carried out with unit variance-scaling (UV) to analyze differences in the metabolite components, taste attributes, and antioxidant activity profiles of the cabbage varieties. OPLS-DA was used to clarify the differences between groups of $x$ variables. Numbers 1 to 6 were assigned to each variety. The data was normalized with UV, and then the discriminant analysis between cabbage varieties was performed using OPLS-DA. OPLS analysis was used to evaluate the correlation between $x$ and $y$ variables in order to identify important $x$ variables that correlate with each $y$ variable by creating a model that predicts $x$ from $y$. The model obtained by OPLS analysis and OPLS-DA was considered to have excellent quantitative and predictive performance for R2Y $\geq 0.65$ and Q2Y $\geq 0.5$ [33]. Model validity and accuracy were assessed using CV-ANOVA and a permutation test. CV-ANOVA is used in OPLS and OPLS-DA significance tests [45]. The significance level was set at $5 \%(p<0.05)$. The permutation test was performed with $n=200$ and evaluated using the R2Y- and 
Q2-intercept. A corresponding regression equation in OPLS was created for any significant model for a given $y$ variable. Additionally, the values of variables important for prediction (VIP) were calculated to identify the characteristic $x$ variables that correlate with each $y$ variable. The VIP values $\geq 1.0$ were considered significant.

\section{Conclusions}

This study correlated the primary metabolites of six cabbage varieties with their characteristic taste and antioxidant activities. Electronic tongue analysis was used to evaluate the nine constituent tastes objectively, while biochemical assays were used to quantitate the antioxidant activities in each variety. As a result, significant predictive models were successfully created for DPPH, sourness, acidic bitterness, irritant, umami, saltiness, bitterness, astringency, and richness. The high-VIP components of each model demarcate characteristic metabolites that show differences between varieties. The metabolites identified in this study as being key factors in cabbage taste and antioxidant activity were 4-aminobutyric acid (DPPH and umami), fructose 1-phosphate (sourness), adipic acid (acidic bitterness), 5-oxoproline (irritant and saltiness), $N$-acetylglycine (bitterness), O-phosphoethanolamine (astringency), and homovanillic acid (richness). Additionally, these represent markers indicating breed differences. The success of this study paves the way for future investigations of secondary plant metabolite involvement in cabbage breed-specific characteristics as well as the examination of the effects of growth conditions on metabolite composition and resultant attributes.

Supplementary Materials: The following are available online. Table S1: List of $x$ and $y$ variables. Table S2: The $p$-values obtained by Tukey's multiple comparison test for antioxidant activity (Figure 1) and taste attributes (Figure 2). Table S3: List of VIP values obtained by OPLS analysis. Figure S1: Typical total ion chromatograms obtained by GC-MS analysis of different cabbage varieties. Figure S2: (A) Score plots and (B) loading plots obtained by PCA-X analysis of cabbage varieties. Figure S3: (A) Score plots and (B) loading plots of OPLS-DA results for the different cabbage varieties.

Author Contributions: Conceptualization, R.M.; Methodology, R.M.; Validation, M.T., C.N., and N.T.; Formal analysis, R.M.; Investigation, M.T. and C.N.; Data curation, M.T. and C.N.; Writing—original draft preparation, R.M.; Writing-review and editing, S.T.; Visualization, R.M., C.N., and N.T.; Supervision, R.M.; Project administration, R.M.; Funding acquisition, R.M.

Funding: This research received no external funding.

Acknowledgments: This work was supported by the Agricultural Promotion Center of Kure City, the Kure Industrial Promotion Center and the Hirokanran Production Association. We thank the Agricultural Promotion Center of Kure City for supplying the cabbage samples. We would like to thank Editage (www.editage.jp) for English language editing.

Conflicts of Interest: The authors declare no conflict of interest.

\section{References}

1. Verkerk, R.; Schreiner, M.; Krumbein, A.; Ciska, E.; Holst, B.; Rowland, I.; De Schrijver, R.; Hansen, M.; Gerhäuser, C.; Mithen, R.; et al. Glucosinolates in Brassica vegetables: The influence of the food supply chain on intake, bioavailability and human health. Mol. Nutr. Food. Res. 2009, 53 (Suppl. 2), S219-S265. [CrossRef]

2. Fahey, J.W.; Zalcmann, A.T.; Talalay, P. The chemical diversity and distribution of glucosinolates and isothiocyanates among plants. Phytochemistry 2001, 56, 5-51. [CrossRef]

3. Brennan, P.; Hsu, C.C.; Moullan, N.; Szeszenia-Dabrowska, N.; Lissowska, J.; Zaridze, D.; Rudnai, P.; Fabianova, E.; Mates, D.; Bencko, V.; et al. Effect of cruciferous vegetables on lung cancer in patients stratified by genetic status: A mendelian randomisation approach. Lancet 2005, 366, 1558-1560. [CrossRef]

4. Onyilagha, J.; Bala, A.; Hallett, R.; Gruber, M.; Soroka, J.; Westcott, N. Leaf flavonoids of the cruciferous species, Camelina sativa, Crambe spp., Thlaspi arvense and several other genera of the family Brassicaceae. Biochem. Syst. Ecol. 2003, 31, 1309-1322. [CrossRef]

5. Vallejo, F.; Gil-Izquierdo, A.; Pérez-Vicente, A.; García-Viguera, C. In vitro gastrointestinal digestion study of broccoli inflorescence phenolic compounds, glucosinolates, and vitamin C. J. Agric. Food Chem. 2004, 52, 135-138. [CrossRef] [PubMed] 
6. Putri, S.P.; Nakayama, Y.; Matsuda, F.; Uchikata, T.; Kobayashi, S.; Matsubara, A.; Fukusaki, E. Current metabolomics: Practical applications. J. Biosci. Bioeng. 2013, 115, 579-589. [CrossRef] [PubMed]

7. Putri, S.P.; Yamamoto, S.; Tsugawa, H.; Fukusaki, E. Current metabolomics: Technological advances. J. Biosci. Bioeng. 2013, 116, 9-16. [CrossRef] [PubMed]

8. Andjelković, U.; Šrajer Gajdošik, M.; Gašo-Sokač, D.; Martinović, T.; Josić, D. Foodomics and food safety: Where we are. Food Technol. Biotechnol. 2017, 55, 290-307. [CrossRef]

9. Witzel, K.; Neugart, S.; Ruppel, S.; Schreiner, M.; Wiesner, M.; Baldermann, S. Recent progress in the use of 'omics technologies in brassicaceous vegetables. Front. Plant Sci. 2015, 6, 244. [CrossRef]

10. Kim, J.; Jung, Y.; Song, B.; Bong, Y.-S.; Ryu, D.H.; Lee, K.-S.; Hwang, G.-S. Discrimination of cabbage (Brassica rapa ssp. pekinensis) cultivars grown in different geographical areas using $1 \mathrm{H}-\mathrm{NMR}-$ based metabolomics. Food Chem. 2013, 137, 68-75. [CrossRef]

11. Jeon, J.; Lim, C.J.; Kim, J.K.; Park, S.U. Comparative metabolic profiling of green and purple pakchoi (Brassica Rapa Subsp. Chinensis). Molecules 2018, 23, 1613. [CrossRef] [PubMed]

12. Wang, Y.-Q.; Hu, L.-P.; Liu, G.-M.; Zhang, D.-S.; He, H.-J. Evaluation of the nutritional quality of Chinese kale (Brassica alboglabra Bailey) using UHPLC-quadrupole-orbitrap MS/MS-based metabolomics. Molecules 2017, 22, 1262. [CrossRef] [PubMed]

13. Baek, S.-A.; Jung, Y.-H.; Lim, S.-H.; Park, S.U.; Kim, J.K. Metabolic profiling in Chinese cabbage (Brassica rapa L. subsp. pekinensis) cultivars reveals that glucosinolate content is correlated with carotenoid content. J. Agric. Food Chem. 2016, 64, 4426-4434. [CrossRef] [PubMed]

14. Mie, A.; Laursen, K.H.; Åberg, K.M.; Forshed, J.; Lindahl, A.; Thorup-Kristensen, K.; Olsson, M.; Knuthsen, P.; Larsen, E.H.; Husted, S. Discrimination of conventional and organic white cabbage from a long-term field trial study using untargeted LC-MS-based metabolomics. Anal. Bioanal. Chem. 2014, 406, 2885-2897. [CrossRef] [PubMed]

15. Hennig, K.; de Vos, R.C.H.; Maliepaard, C.; Dekker, M.; Verkerk, R.; Bonnema, G. A metabolomics approach to identify factors influencing glucosinolate thermal degradation rates in Brassica vegetables. Food Chem. 2014, 155, 287-297. [CrossRef] [PubMed]

16. Abdel-Farid, I.B.; Kim, H.K.; Choi, Y.H.; Verpoorte, R. Metabolic characterization of Brassica rapa leaves by NMR spectroscopy. J. Agric. Food Chem. 2007, 55, 7936-7943. [CrossRef]

17. Park, S.-Y.; Lim, S.-H.; Ha, S.-H.; Yeo, Y.; Park, W.T.; Kwon, D.Y.; Park, S.U.; Kim, J.K. Metabolite profiling approach reveals the interface of primary and secondary metabolism in colored cauliflowers (Brassica oleracea L. ssp. botrytis). J. Agric. Food Chem. 2013, 61, 6999-7007. [CrossRef]

18. Moyo, M.; Amoo, S.O.; Aremu, A.O.; Gruz, J.; Šubrtová, M.; Jarošová, M.; Tarkowski, P.; Doležal, K. Determination of mineral constituents, phytochemicals and antioxidant qualities of Cleome gynandra, compared to Brassica oleracea and Beta vulgaris. Front. Chem. 2018, 5, 128. [CrossRef]

19. Li, Z.; Lee, H.W.; Liang, X.; Liang, D.; Wang, Q.; Huang, D.; Ong, C.N. Profiling of phenolic compounds and antioxidant activity of 12 cruciferous vegetables. Molecules 2018, 23, 1139. [CrossRef]

20. Karadag, A.; Ozcelik, B.; Saner, S. Review of methods to determine antioxidant capacities. Food Anal. Methods 2009, 2, 41-60. [CrossRef]

21. Shahidi, F.; Zhong, Y. Measurement of antioxidant activity. J. Funct. Foods 2015, 18, 757-781. [CrossRef]

22. Roy, M.K.; Koide, M.; Rao, T.P.; Okubo, T.; Ogasawara, Y.; Juneja, L.R. ORAC and DPPH assay comparison to assess antioxidant capacity of tea infusions: Relationship between total polyphenol and individual catechin content. Int. J. Food Sci. Nutr. 2010, 61, 109-124. [CrossRef] [PubMed]

23. Tahara, Y.; Toko, K. Electronic tongues-A review. IEEE Sensors J. 2013, 13, 3001-3011. [CrossRef]

24. Scampicchio, M.; Ballabio, D.; Arecchi, A.; Cosio, S.M.; Mannino, S. Amperometric electronic tongue for food analysis. Microchim. Acta 2008, 163, 11-21. [CrossRef]

25. Podrażka, M.; Bączyńska, E.; Kundys, M.; Jeleń, P.S.; Witkowska Nery, E. Electronic tongue-A tool for all tastes? Biosensors 2018, 8, 3. [CrossRef] [PubMed]

26. Makino, Y.; Hashizume, M.; Boerzhijin, S.; Akihiro, T.; Yamada, T.; Okazaki, K. Influence of cold or frozen storage on temporal changes in sulforaphane and objective taste values of broccoli (Brassica oleracea var. italica) florets. Environ. Control Biol. 2019, 57, 45-51. [CrossRef]

27. Chen, W.; Taylor, M.C.; Barrow, R.A.; Croyal, M.; Masle, J. Loss of phosphoethanolamine N-methyltransferases abolishes phosphatidylcholine synthesis and is lethal. Plant Physiol. 2019, 179, 124-142. [CrossRef] 
28. Park, S.; Arasu, M.V.; Lee, M.-K.; Chun, J.-H.; Seo, J.M.; Lee, S.-W.; Al-Dhabi, N.A.; Kim, S.-J. Quantification of glucosinolates, anthocyanins, free amino acids, and vitamin $\mathrm{C}$ in inbred lines of cabbage (Brassica oleracea L.). Food Chem. 2014, 145, 77-85. [CrossRef]

29. Wieczorek, M.N.; Walczak, M.; Skrzypczak-Zielińska, M.; Jeleń, H.H. Bitter taste of Brassica vegetables: The role of genetic factors, receptors, isothiocyanates, glucosinolates, and flavor context. Crit. Rev. Food Sci. Nutr. 2018, 58, 3130-3140. [CrossRef]

30. Sawai, Y.; Moon, J.-H. NMR analytical approach to clarify the molecular mechanisms of the antioxidative and radical-scavenging activities of antioxidants in tea using 1,1-diphenyl-2-picrylhydrazyl. J. Agric. Food Chem. 2000, 48, 6247-6253. [CrossRef]

31. Sawai, Y.; Moon, J.-H.; Sakata, K.; Watanabe, N. Effects of structure on radical-scavenging abilities and antioxidative activities of tea polyphenols: NMR analytical approach using 1,1-diphenyl-2-picrylhydrazyl radicals. J. Agric. Food Chem. 2005, 53, 3598-3604. [CrossRef] [PubMed]

32. Villaño, D.; Fernández-Pachón, M.S.; Moyá, M.L.; Troncoso, A.M.; García-Parrilla, M.C. Radical scavenging ability of polyphenolic compounds towards DPPH free radical. Talanta 2007, 71, 230-235. [CrossRef] [PubMed]

33. Eriksson, L.; Byrne, T.; Johansson, E.; Trygg, J.; Vikström, C. Multi- and Megavariate Data Analysis, Basic Principles and Applications, 3rd ed.; MKS Umetrics AB: Malmö, Sweden, 2013.

34. Azizan, A.; Ahamad Bustamam, M.S.; Maulidiani, M.; Shaari, K.; Ismail, I.S.; Nagao, N.; Abas, F. Metabolite profiling of the microalgal diatom Chaetoceros calcitrans and correlation with antioxidant and nitric oxide inhibitory activities via ${ }^{1} \mathrm{H}-\mathrm{NMR}-$ based metabolomics. Mar. Drugs 2018, 16, 154. [CrossRef] [PubMed]

35. Bottone, A.; Montoro, P.; Masullo, M.; Pizza, C.; Piacente, S. Metabolomics and antioxidant activity of the leaves of Prunus dulcis Mill. (Italian cvs. Toritto and Avola). J. Pharm. Biomed. Anal. 2018, 158, 54-65. [CrossRef] [PubMed]

36. Lee, S.Y.; Mediani, A.; Ismail, I.S.; Maulidiani, H.; Abas, F. Antioxidants and $\alpha$-glucosidase inhibitors from Neptunia oleracea fractions using ${ }^{1} \mathrm{H}$ NMR-based metabolomics approach and UHPLC-MS/MS analysis. BMC Complement. Altern. Med. 2019, 19, 7. [CrossRef] [PubMed]

37. Sugimoto, M.; Koseki, T.; Hirayama, A.; Abe, S.; Sano, T.; Tomita, M.; Soga, T. Correlation between sensory evaluation scores of Japanese sake and metabolome profiles. J. Agric. Food Chem. 2010, 58, 374-383. [CrossRef] [PubMed]

38. Várvölgyi, E.; Gere, A.; Szöllősi, D.; Sipos, L.; Kovács, Z.; Kókai, Z.; Csóka, M.; Mednyánszky, Zs.; Fekete, A.; Korány, K. Application of sensory assessment, electronic tongue and GC-MS to characterize coffee samples. Arab. J. Sci. Eng. 2015, 40, 125-133. [CrossRef]

39. Mabuchi, R.; Ishimaru, A.; Tanaka, M.; Kawaguchi, O.; Tanimoto, S. Metabolic profiling of fish meat by GC-MS analysis, and correlations with taste attributes obtained using an electronic tongue. Metabolites 2019, 9, 1. [CrossRef]

40. Mabuchi, R.; Ishimaru, A.; Adachi, M.; Zhao, H.; Kikutani, H.; Tanimoto, S. Taste evaluation of yellowtail (Seriola quinqueradiata) ordinary and dark muscle by metabolic profiling. Molecules 2019, 24, 2574. [CrossRef]

41. Mabuchi, R.; Adachi, M.; Kikutani, H.; Tanimoto, S. Discriminant analysis of muscle tissue type in yellowtail Seriola quinqueradiata muscle based on metabolic component profiles. Food Sci. Technol. Res. 2018, 24, 883-891. [CrossRef]

42. Katsube, T.; Tabata, H.; Ohta, Y.; Yamasaki, Y.; Anuurad, E.; Shiwaku, K.; Yamane, Y. Screening for antioxidant activity in edible plant products: Comparison of low-density lipoprotein oxidation assay, DPPH radical scavenging assay, and Folin-Ciocalteu assay. J. Agric. Food Chem. 2004, 52, 2391-2396. [CrossRef] [PubMed]

43. Prior, R.L.; Hoang, H.; Gu, L.; Wu, X.; Bacchiocca, M.; Howard, L.; Hampsch-Woodill, M.; Huang, D.; Ou, B.; Jacob, R. Assays for hydrophilic and lipophilic antioxidant capacity (oxygen radical absorbance capacity $\left.\left(\mathrm{ORAC}_{\mathrm{FL}}\right)\right)$ of plasma and other biological and food samples. J. Agric. Food Chem. 2003, 51, 3273-3279. [CrossRef] [PubMed]

44. Anjiki, N.; Hosoe, J.; Fuchino, H.; Kiuchi, F.; Sekita, S.; Ikezaki, H.; Mikage, M.; Kawahara, N.; Goda, Y. Evaluation of the taste of crude drug and Kampo formula by a taste-sensing system (4): Taste of processed aconite root. J. Nat. Med. 2011, 65, 293-300. [CrossRef] [PubMed] 
45. Eriksson, L.; Trygg, J.; Wold, S. CV-ANOVA for significance testing of PLS and OPLS ${ }^{\circledR}$ models. J. Chemom. 2008, 22, 594-600. [CrossRef]

Sample Availability: Not available.

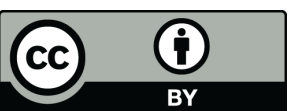

(C) 2019 by the authors. Licensee MDPI, Basel, Switzerland. This article is an open access article distributed under the terms and conditions of the Creative Commons Attribution (CC BY) license (http://creativecommons.org/licenses/by/4.0/). 